\title{
Hysteresis Thresholding: A Graph-Based Wavelet Block Denoising Algorithm
}

\author{
Radu Ranta* and Valérie Louis-Dorr
} Centre de Recherche en Automatique de Nancy (CRAN), Nancy-Université, CNRS, 2 avenue de la Forêt de Haye
F-54516 Vandoeuvre-les-Nancy, France

\begin{abstract}
This communication aims to combine several previously proposed wavelet denoising algorithms into a novel heuristic block method. The proposed "hysteresis" thresholding uses two thresholds simultaneously in order to combine detection and minimal alteration of informative features of the processed signal. This approach exploits the graph structure of the wavelet decomposition to detect clusters of significant wavelet coefficients. The new algorithm is compared with classical denoising methods on simulated benchmark signals.
\end{abstract}

Keywords: Wavelets, block denoising, graphs, transitive closure.

\section{INTRODUCTION}

One of the most important applications of wavelets is the signal denoising. The fundamental hypothesis is that wavelets are correlated with the informative signal and uncorrelated with the noise, which globally means that large absolute value coefficients correspond to signal and small coefficients to noise. Therefore, noise cancelling (as well as compression) can be performed by thresholding: small coefficients will be discarded and the few remaining large magnitude coefficients will be used to reconstruct the informative signal. Several approaches were proposed since the pioneering works of Donoho et al. [1,2] (see Antoniadis et al. for a comprehensive review [3, 4]). In this communication, we propose to combine previously introduced thresholds in a novel block-denoising approach that takes into account the local wavelet graph structure.

This paper is organized as follows. In the next section, we recall the most critical issues in wavelet denoising and some of the proposed solutions, emphasizing the methods at the origin of our work. Section 3 introduces the graph-based approach and the new "hysteresis" thresholding. The fourth section presents and discusses the simulation results, comparing them with classic denoising algorithms, and it is followed by a short conclusion.

\section{WAVELET DENOISING}

We consider the model $z=x+n$, where $z$ is the noisy discrete-time signal (length $N$ ), $x$ is the noise-free unknown version of $z$ and $n$ the noise. Synthetically, the discrete orthogonal wavelet transform (DWT) of $z$ writes:

$$
z=\sum_{p, j} w_{z}^{j, p} \psi^{j, p}+\sum_{p} w_{z}^{M, p} \phi^{M, p}
$$

*Address correspondence to this author at the Centre de Recherche en Automatique de Nancy (CRAN), Nancy-Université, CNRS, 2 avenue de la Forêt de Haye F-54516 Vandoeuvre-les-Nancy, France;

E-mail: radu.ranta@ensem.inpl-nancy.fr where $j=[1 . . M]$ is the scale, $p=\left[1 . .2^{-M} N\right]$ the position, $\psi$ the wavelet, $\phi$ the scaling function and $M$ is the analysis depth [5]. The wavelet coefficients vectors of $z, x$ and $n$ are related by:

$$
w_{z}=w_{x}+w_{n}
$$

The denoised signal's coefficients vector $w_{x}$ is estimated by modifying $w_{z}=\left[w_{z}^{M, p} w_{z}^{j, p}\right]$. Generally, the approximation coefficients $w_{z}^{M, p}$ remain unchanged.

Two main families of approaches are proposed in the literature: term-by-term and block approaches. In the former, the estimated coefficients $w_{x}$ are given by:

$\hat{w}_{x}=g\left(w_{z}\right) w_{z}$,

with $g\left(w_{z}\right)$ a shrinkage function applied on the measured signal coefficients. In the case of block thresholding, the shrinkage function depends not only on the currently evaluated coefficient, but also on a group or block of neighboring coefficients $w_{z, b}$ (the $b$ subscript indicates the adjacent coefficients from the block $B$ ). Moreover, the shrinkage can be applied for the whole considered block or for a given subset $w_{z, b 1} \subset w_{z, b}$. In practice [6], the size of block is 3 and $w_{z, b 1}$ is the middle element:

$w_{x, b 1}=g\left(w_{z, b}\right) w_{z, b 1}$.

Several algorithms from these two families are briefly presented next. They are integrated in the novel hysteresis algorithm and/or used for comparison.

\subsection{Term-by-Term Shrinkage}

As the noise-free coefficient vector is presumably sparse, the simplest solution is provided by the well-known hard shrinkage (thresholding): 


$$
g\left(w_{z}\right)=\max \left(0, \operatorname{sign}\left(\left|w_{z}\right|-T\right)\right),
$$

where $T$ is a threshold value. The most widely used is the universal threshold $T_{U}$ proposed by Donoho and Johnstone in their algorithm VisuShrink (designated Visu in the sequel) [1]. Visu is used to achieve complete asymptotic elimination of the normal Gaussian noise and it can be shown (using extreme values statistics) that this is achieved by setting $T_{U}=\sqrt{2 \log N}$. This method is very appealing because of its simplicity and its visually attractive results, however, its focus on eliminating all noise often leads to less precise reconstruction of the signal of interest and that represents a major drawback.

A different approach is proposed by the Sure algorithm [2], that aims to estimate as precisely as possible the "clean" signal by minimizing an estimate of the mean squared error (MSE) between the denoised signal and the original one, known as the Stein Unbiased Risk Estimator. This risk is minimized by exhaustively searching the optimal threshold among the coefficients $w_{z}$. The algorithm was developed under specific conditions on the function $g$ (weak differentiability). In particular, the most widely used is the soft thresholding function:

$$
g\left(w_{z}\right)=\max \left(0, \frac{\left|w_{z}\right|-T}{\left|w_{z}\right|}\right)
$$

The obtained threshold $T_{S}$ (or thresholds, as the method is usually implemented by scale) are lower than the $T_{U}$ and the obtained signal has a noisier appearance.

The iterative method we proposed in [7,8] follows a different approach, inspired by [9-11]: the threshold $T_{M}$ is obtained by a fast fixed-point parameter-free algorithm based on a generalized Gaussian (GG) modelling of the wavelet coefficients. The resulting "minimal denoising", called hereafter $\operatorname{Min} D$, ensures robust detection of high value coefficients (outliers) and thus leads to maximum information extraction from the measured signal.

As the universal threshold proposed in the Visu algorithm, the threshold computed by $\operatorname{Min} D$ does not impose specific characteristics on the function $g($.$) .$ Therefore, both methods can be applied either with hard (5) or soft (6) shrinkage.

\subsection{Block Shrinkage}

A quite natural hypothesis, most of the times verified in applications, is that large wavelet coefficients appear in clusters, or blocks. The approaches introduced by Hall, Kerkyacharian and Picard [12] and Cai et al. [6, 13, 14] aim to take into account local features of the signal by adapting thresholds to blocks of neighboring coefficients. The most well-known methods are BlockJS (BJS) and NeighBlock $(N B)$, with its simpler version NeighCoef $[6,13]$. The two algorithms propose a similar philosophy as Sure: estimate as precisely as possible a signal corrupted by noise by minimizing the James-Stein estimate of the MSE risk. This minimization leads to a particular form of the shrinkage function $g($.$) , which applies to all coefficients w_{z, b}$ in a given block of size $L$ :

$g\left(w_{z, b}\right)=\max \left(0, \frac{\left\|w_{z, b}\right\|^{2}-T_{L}}{\left\|w_{z, b}\right\|^{2}}\right)$,

with $\left\|x_{b}\right\|^{2}$ the energy of the block and $T_{L}$ a block-size depending threshold. For $B J S, T_{L}=\lambda L \sigma^{2}$, with $\lambda=4.505 \ldots, L=\log N$ ( $N$ being the signal length) and $\sigma$ the estimated noise power (by scale or global, for colored and white noise respectively). The difference between $B J S$ and $N B$ consists in the use of the function $g($.$) : while in$ $B J S$ all coefficients in the block are shrunk by the same amount $\left(g\left(w_{z, b}\right)\right)$, in $N B$ this is done only for the $L / 2$ coefficients located in the center of the considered block. In this case, the blocks will not be disjoints but overlapped by $L / 4$ (see [6] for more details). The neighborhood proposed in these approaches is constructed scale-by-scale, without inter-scale dependencies. Extensions taking into account this last item were proposed recently by $[15,16]^{1}$.

\section{GRAPH-BASED HYSTERESIS APPROACH}

Most of the wavelet denoising algorithms treat the coefficients either individually or in blocks grouped by scale. These approaches don't take into account more general neighborhoods, spanned over scales as well as in time. This point is easily captured by the graph structure of the DWT.

\subsection{Graph Structures}

Currently, the most complete investigation of the graph structure of the DWT, implicitly addressing both inter- and intra-scale relations between coefficients, is done by Crouse et al. [20]. The authors, who attach a Hidden Markov Model (HMM) to the graph to compute the thresholds, propose three different architectures and thus types of neighborhoods: the Independent Mixture IM (i.e., the neighboring coefficients are not considered in the procedure), the Hidden Markov Chain HMC (i.e., considering dependencies by scale) and the Hidden Markov Tree HMT (binary trees resulting from the wavelet decomposition). A graphical representation is given Fig. (1).

An implicit advantage of the graph representation, evidenced by [20], is that it emphasizes two important properties of real signals, persistence and clustering. Persistence implies that "large/small values of wavelet coefficients tend to propagate across scales", which means that the binary trees of wavelet coefficients (Fig. 1a) tend to contain similar amplitude coefficients. Therefore, if a high value coefficients is selected by a thresholding algorithm, it is very likely that the the connected coefficients in the graph, i.e., belonging to the same tree, should be selected also. Clustering, defined as the fact that "if a particular wavelet coefficients is large/small, then adjacent coefficients are very likely to also be large/small", considers that connected

${ }^{1}$ Bayesian block denoising methods (for example [17-19]) are not treated here. 


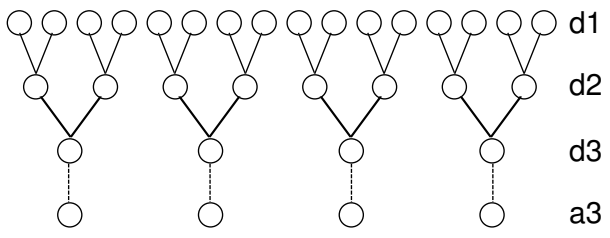

(a) a-o-o-o-o-o-o-o-o-o-o-o d1

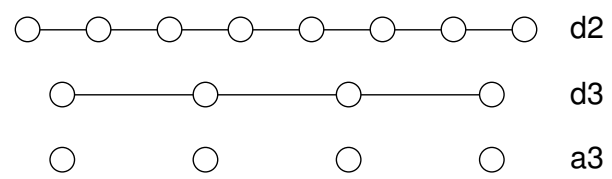

(b)

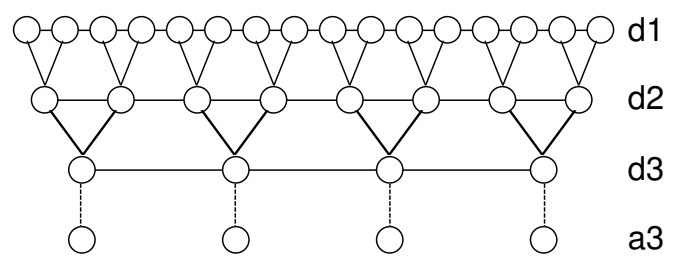

(c)

Fig. (1). Graph types: (a) tree; (b) scale; (c) complete.

neighboring coefficients belonging to the same scale should be selected together if one of them is superior to the threshold (Fig. 1b). If both persistence and clustering are considered, all the coefficients are linked together in a complete graph (Fig. 1c).

A formal representation of graphs is given by the adjacency matrix $A$. For a graph having $N$ nodes (wavelet coefficients), $A$ is a square $N \times N$ boolean matrix with elements $A(i, j)=1$ if there is an edge between nodes $i$ and $j$ and 0 elsewhere. The obtained matrix is symmetric, and the non-zero elements on line (column) $i$ correspond to the coefficients connected to element $w_{z}(i)$ of the wavelet coefficients vector $w_{z}$. By convention, we consider every node connected to itself (the diagonal elements $A(i, i)=1$ ).

\subsection{Pruned Graphs}

The three term-by-term denoising methods presented in the previous section have different rationales: the universal thresholding Visu uses a high threshold aiming to select only informative coefficients and eliminate all noise, while Sure and MinD use a low threshold to eliminate only noise and therefore to select all the signal. A heuristic combination can be then proposed: use a Visu computed high threshold $T_{h}=T_{U}$ to select blocks of significant wavelet coefficients, and a Sure or MinD low threshold $T_{l}=T_{S}\left(T_{M}\right)$ to fix the limits of the selected blocks. In the graph structure, the neighborhood of a "very large" coefficient $\left|w_{z}\right|>T_{h}$ selected by Visu will be formed by the connected "large enough" coefficients selected by Sure or $\operatorname{MinD}\left(\left|w_{z}\right|>T_{l}\right)$. These coefficients are called further-on max-selected and minselected respectively. The resulting blocks, without a priori predefined shapes, will naturally integrate both persistence and clustering properties of real signals.
The previous approach was implemented in a three-step algorithm, illustrated also in Fig. (2).

1. For the considered graph adjacency matrix $A$ (corresponding to graph 1), keep only the 1's corresponding to min-selected elements (marked nodes) by forcing other lines and columns to 0 :

$A(i,)=.A(., i)=0, \forall\left|w_{z}(i)\right|<T_{l}$

Note the newly obtained adjacency matrix $A_{1}$. The resulting graph (Fig. 2b) is a collection of disjoint blocks (clusters) of min-selected coefficients. For a given cluster, we note $B$ the set of the indices $b$ of the coefficients belonging to it, $\left|w_{z}(b)\right| \geq T_{l}$.

2. Apply a transitive closure procedure on the pruned graph: if there is a path between two nodes add a new edge between them. This approach leads to an iterative computation of a new adjacency matrix, known as Warshall's algorithm [21]: at each step $l$,

$A_{1, l}(\alpha, \beta)=A_{1, l-1}(\alpha, \beta) \vee\left(A_{1, l-1}(\alpha, \gamma) \wedge A_{1, l-1}(\gamma, \beta)\right)$

At convergence, all the nodes in a cluster $B$ will be connected by an edge (Fig. 2c). Consequently, if the nodes $b_{i}$ and $b_{j}$ belong to the cluster $B$, the elements of the obtained adjacency matrix $A_{2}$ are $A_{2}\left(b_{i}, b_{j}\right)=A_{2}\left(b_{j}, b_{i}\right)$ $=1, \forall b_{i}, b_{j} \in B$. An implementation issue must be noted here: a complete iterative transitive closure (9) may lead to a very slow and memory consuming algorithm, especially for long and not very sparse signals (large $N$ ). This problem depends on the chosen graph structure: tree-type graphs (Fig. 1a) have a limited number of possible connections depending on the wavelet decomposition depth, while scaletype (Fig. 1b) or complete graphs (Fig. 1c) are only limited 


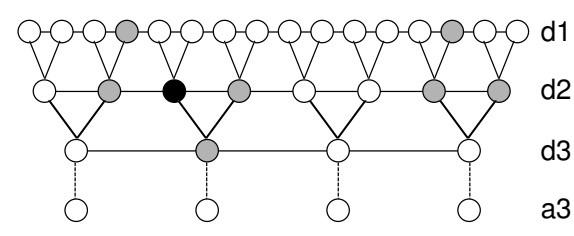

(a)

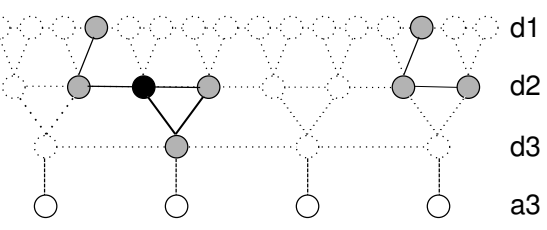

(b)

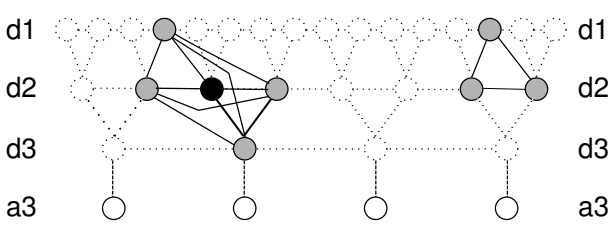

(c)

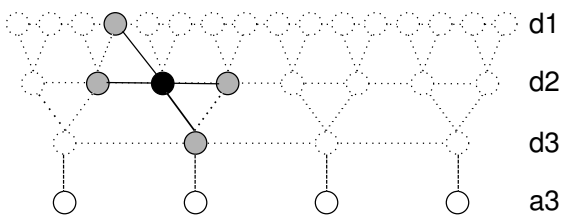

(d)

Fig. (2). Pruning algorithm: (a) Original graph: max-selected and min-selected coefficients are figured in black and gray respectively; (b) First pruning: discard the small values; (c) Transitive closure; (d) Second pruning and final result. The approximation scale is not affected.

by the length of the signal. Therefore, the maximum number of iterations should be limited by the user. We propose here to limit them to the maximum number of levels of the wavelet decomposition;

3. Construct a final adjacency matrix $A_{3}$ by selecting the blocks containing at least one max-selected coefficient, i.e., only the lines (columns) $i$ of $A_{2}$ corresponding to the coefficients $\left|w_{z}(i)\right| \geq T_{h}$ (Fig. 2d):

$$
A_{2}(i, .)=A_{2}(., i)=0, \forall\left|w_{z}(i)\right|<T_{h} \text {. }
$$

The non-null lines (columns) of $A_{3}$ correspond to the wavelet coefficients used for reconstruction.

The final denoising algorithm will have the following structure:

1. Wavelet transform the measured signal.

2. Choose the graph type: scale, tree or complete.

3. Apply Sure or MinD, global or scale by scale, to mark min-selected coefficients and the corresponding nodes of the graph.

4. Apply Visu to mark max-selected coefficients and nodes.

5. Prune according to the given procedure the chosen graph.

6. Reconstruct by inverse wavelet transform.

\section{RESULTS AND DISCUSSION}

Several versions of the hysteresis approach were implemented:
- considering either Sure (noted HyVS) or MinD (noted $H y V M$ ) algorithms for the low threshold, and Visu for the high threshold;

- $\quad$ considering the three types of graph-structures: by scale, subscript $s$, by binary tree, subscript $t$ and the complete graph, subscript $c$;

- $\quad$ adapted to white or colored noise (white: the noise power is estimated on the first details level for Visu and Sure, fixed point iterations are performed on all coefficients for MinD ; colored: scale by scale noise estimation for Visu and Sure, by-scale fixed-point descent for $\operatorname{Min} D$ );

- $\quad$ considering soft (6) or hard (5) thresholding.

The algorithms were compared using the mean squared error MSE to classical term-by-term Visu, Sure and $\operatorname{MinD}$, as well as to the block methods $B J S$ and $N B$, adapted from the scripts of the free toolbox of Antoniadis $e t$ al. [22].

Concerning the thresholding strategy, it must be mentioned here that Sure, BJS and $N B$ were specifically developed for soft-type shrinkage functions $g($.), while Visu and MinD only compute threshold values and the user can choose the shrinkage strategy. The results presented here in details concern only the soft thresholding, the low threshold value given by Sure or by $\operatorname{Min} D$ being subtracted from the selected coefficients. Hard thresholding particularities will be briefly exposed at the end of the section. The approximation scale was kept unchanged, although for sparse signals the results might be improved if the approximation is thresholded also. 
The tested signals were the usual Bumps, Blocks, HeaviSine and Doppler [1], with lengths from 512 to 16384. Gaussian noise was added, with signal to noise ratios (SNR) of 3, 5 and 7. Three types of noise were used: white, low-pass filtered ( $4^{\text {th }}$ order Butterworth filter, normalized cut-off frequency at 0.25 ) and high-pass filtered ( $4^{\text {th }}$ order Butterworth filter, normalized cut-off frequency at 0.25 ).

Twenty simulations were performed for each combination of signal type, length and SNR. For the white noise case, the most significant numerical results are presented in Table 1. These results are averaged by signal type, signal length and signal to noise ratio, in order to illustrate independently the influence of these parameters on the algorithms' performances.

As it can be seen Table 1, the signal type has a certain but limited influence on the performances: for the first 2 signals (Blocks and Bumps), the novel hysteresis approach based on universal and SURE thresholds and applied on a complete-type graph $\left(H y V S_{c}\right)$ performs slightly better than Sure. The other two graph types (scale $H y V S_{s}$ and tree $H y V S_{t}$ ) follow closely, with a rather important gap between them and the block-approaches BJS and $N B$. On Heavisine, all hysteresis type approaches perform better than the other algorithms ( $H y V M_{c}$ included), while Sure shows the worst performances, MinD being better than the other term-by-term thresholding methods, as well as than the tested block approaches. On the contrary, on the frequency rich Doppler, BJS shows the best performance, followed by the three HyVS algorithms. The ratios of improvement vary: $H y V S_{c}$ outperforms Sure and BJS by mean squares errors smaller by 8 to $40 \%$ for Bumps, Blocks and HeaviSine while BJS is better by about $8 \%$ for the Doppler signal.
The signal length $N$ plays an important role in the algorithms performances. All of them improve when $N$ increases, the amelioration being more marked for asymptotic procedures as Visu and for probability density estimation methods like MinD. Globally, for the three graph types, the HyVS algorithm shows very good performances regardless of the signal length, compared to the others, with the complete graph approach $H y V S_{c}$ being constantly the most efficient. Sure is the next in line, followed by the block-thresholding methods $B J S$ and $N B$. For the longest tested signals $(N=16386)$, minimal denoising based methods $H y V M_{c}$ and MinD outperform these last two algorithms $B J S$ and $N B$. On the other hand, the computational burden and thus the execution time increases differently with $N$. If for 2048 samples signals, $H y V S_{c}$ and $H y V M_{c}$ are slightly faster than BJS (but slower than Sure), for 16386 length signals the difference becomes important: while $B J S$ is only 1.5 times slower, both hysteresis methods slow their execution about 40 times. This augmentation is due to the fact that complete $N \times N$ graph adjacency matrices (although sparse) are stored and manipulated in our implementation, but more optimal programming solutions can be found by taking into account the proposed bound for the block size (depth of the wavelet decomposition).

As for the influence of the noise power, all algorithms are affected in the same way and their order remains generally unchanged: $H y V S_{c}$ shows constantly the best performances, while the other algorithms from the same family (tree and scale based graphs) and Sure come next.

For the low-frequency and high-frequency noise cases, we tested versions of the described algorithms adapted to colored noise, i.e., scale by scale estimation of $\sigma$ for Visu and Sure based methods, respectively scale by scale probability density estimation and thresholding for MinD

Table 1. MSE for white Noise Case Comparison. The Results are Displayed for the Best Versions of the Hysteresis Algorithm

\begin{tabular}{|c|c|c|c|c|c|c|c|c|c|c|}
\hline & & Visu & Sure & $M i n D$ & BJS & $N B$ & $H y V S_{s}$ & $H y V S_{t}$ & $H y V S_{c}$ & $H y V M_{c}$ \\
\hline \multirow[t]{4}{*}{ Signal } & Blocks & 0.4786 & 0.1999 & 0.4435 & 0.3051 & 0.3313 & 0.2304 & 0.2030 & 0.1852 & 0.4465 \\
\hline & Bumps & 0.6512 & 0.1772 & 0.6400 & 0.2460 & 0.2904 & 0.1790 & 0.1772 & 0.1615 & 0.6399 \\
\hline & Heavisine & 0.0437 & 0.0450 & 0.0421 & 0.0433 & 0.0443 & 0.0408 & 0.0399 & 0.0396 & 0.0410 \\
\hline & Doppler & 0.1596 & 0.0797 & 0.1479 & 0.0618 & 0.0752 & 0.0665 & 0.0731 & 0.0663 & 0.1473 \\
\hline \multirow[t]{6}{*}{ Length } & 512 & 0.8813 & 0.2721 & 0.9266 & 0.4062 & 0.4564 & 0.2920 & 0.2730 & 0.2527 & 0.9275 \\
\hline & 1024 & 0.5192 & 0.1866 & 0.4973 & 0.2304 & 0.2630 & 0.1914 & 0.1797 & 0.1657 & 0.4980 \\
\hline & 2048 & 0.2988 & 0.1209 & 0.2516 & 0.1414 & 0.1750 & 0.1223 & 0.1173 & 0.1057 & 0.2534 \\
\hline & 4096 & 0.1580 & 0.0783 & 0.1226 & 0.0975 & 0.1015 & 0.0755 & 0.0750 & 0.0674 & 0.1230 \\
\hline & 8192 & 0.0894 & 0.0543 & 0.0682 & 0.0641 & 0.0689 & 0.0538 & 0.0550 & 0.0494 & 0.0680 \\
\hline & 16386 & 0.0530 & 0.0405 & 0.0440 & 0.0447 & 0.0471 & 0.0400 & 0.0399 & 0.0380 & 0.0423 \\
\hline \multirow[t]{3}{*}{ SNR } & 3 & 0.5311 & 0.2238 & 0.4829 & 0.2942 & 0.3269 & 0.2335 & 0.2216 & 0.2031 & 0.4835 \\
\hline & 5 & 0.2857 & 0.0974 & 0.2825 & 0.1277 & 0.1461 & 0.0997 & 0.0953 & 0.0874 & 0.2828 \\
\hline & 7 & 0.1831 & 0.0551 & 0.1897 & 0.0702 & 0.0829 & 0.0544 & 0.0531 & 0.0490 & 0.1898 \\
\hline
\end{tabular}


based methods. The relative performances of the algorithms are quite similar most of the time, therefore the complete numerical results are not given here. Still, some particular points must be outlined:

- the performances of minimal denoising based algorithms MinD and HyVM degrade sharply when the signal length $N$ is low, as the estimation of the generalized Gaussian (GG) parameters becomes problematic. Therefore, their use is not recommended, unless the signal length is important and/or the coefficient distribution is close to a GG;

- for low-frequency noise, scale adapted denoising tend to do more harm than good: the noise power might be over-estimated on the low frequency scales and the resulting thresholds might be too high, degrading the signal. This is especially true when the SNR is low: the algorithms using a unique noise estimation perform better than the scale-adapted ones.

Finally, we should note that the comparative results obtained for hard-thresholding confirm the better performances of the hysteresis algorithms. As expected, the main challengers among term by term thresholding are Visu and MinD, Sure being designed for soft-thresholding. Among block denoising approaches, both $B J S$ and $N B$ perform better than term-by-term methods, but they are outperformed by hysteresis implementations, either HyVS or HyVM . Between these two families, Sure based HyVS gives better results than minimal denoising based $H y V M$.

Denoising examples for the best algorithm of each family (term-by-term, classical block-thresholding and hysteresis thresholding) are given Fig. (3).

\section{CONCLUSION AND FUTURE RESEARCH}

In this paper, we present a new heuristic method for wavelet denoising. Our approach, which can be considered a block-denoising type method, is based on the graph structure of the orthogonal wavelet decomposition. We propose to combine previously developed algorithms to modify the graph structure of the wavelet decomposition. This two thresholds approach, called "hysteresis denoising", aims to take into account the property of real signals to have wavelet coefficients grouped in clusters, spanned both over time and over scales. Therefore, a high valued threshold is implemented to select only informative signal coefficients, i.e., to eliminate asymptotically all the noise, while a low threshold is used to define neighborhoods, or clusters, of the selected coefficients in which the signal of interest is accurately estimated. The newly proposed method is compared successfully with other wavelet denoising algorithms, which it outperforms most of the time.

By its nature, the hysteresis thresholding is adapted to the detection and the denoising of isolated transients in long
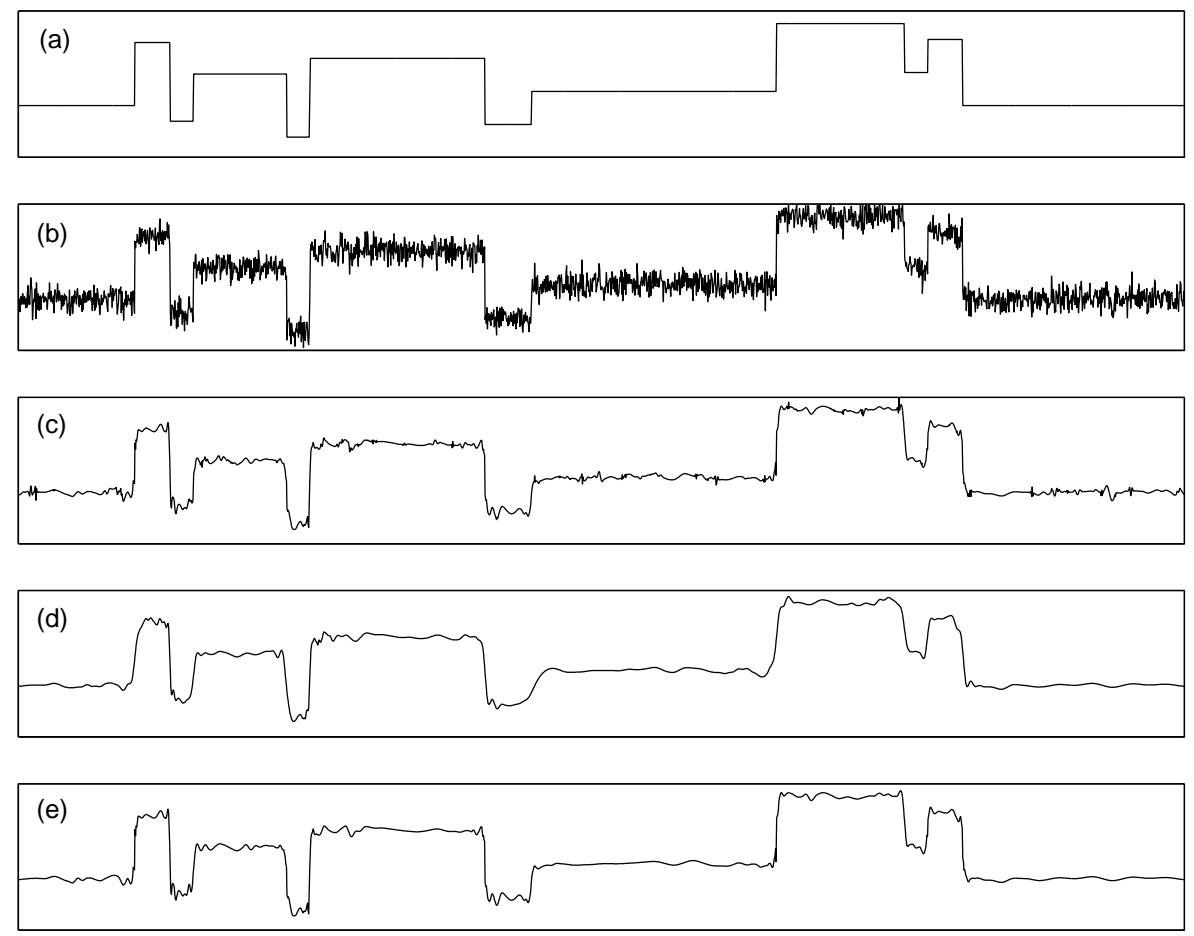

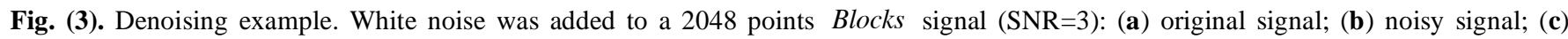

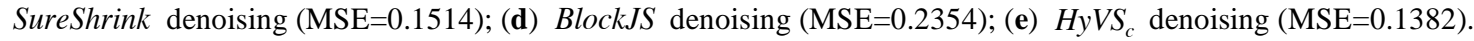


time series, i.e., very sparse signals. The validation of this hypothesis, along with some new improvements on the minimal denoising method, itself adapted to transient detection, and with more extensive comparisons, for example with the recently proposed SureBlock shrinkage [14], which shows rather similar results, are currently under study and will make the subject of a future work.

\section{APPENDIX}

The proposed hysteresis approach is based on the following thresholds:

- high threshold ( Visu universal threshold):

$T_{H}=T_{U}=\sigma \sqrt{2 \log N}$,

with $N$ the signal length and $\sigma$ the noise' (robust) standard deviation;

- low threshold for HyVS (Sure threshold):

$T_{L}=T_{S}=\arg \min _{T \in\left\{w_{z}(k)\right\}}\left(N \sigma^{2}+T^{2} P+\sum_{\substack{k, \mid w_{z}(k)<T}}\left[w_{z}(k)^{2}\right]-2(N-P) \sigma^{2}\right)$

with $P$ the number of wavelet coefficients having absolute values $\geq T$;

- low threshold for $H y V M$ ( $M i n D$ threshold) $T_{L}=T_{M}$ is obtained from the fixed-point iteration:

$$
\begin{aligned}
& T_{i+1}=F_{a m} \sqrt{\frac{1}{N} \sum_{k}\left[w_{z}(k) \cdot \max \left(0, \operatorname{sign}\left(\left|w_{z}(k)\right|-T_{i}\right)\right)\right]^{2}}, \\
& \text { with } F_{a m}=\sqrt{\frac{3 \Gamma\left(\frac{1}{u}\right)}{u}(u e)^{\frac{1}{u}}}
\end{aligned}
$$

[14] T. Cai, and H. Zhou, "A data-driven block thresholding approach to wavelet estimation," The Annals of Statistics, vol. 37, pp. 569-595, 2009.

[15] L. Zhen, H. Zhengjia, Z. Yanyang, and W. Yanxue, "Customized, wavelet denoising using intra- and inter-scale dependency for bearing fault detection," Journal of Sound and Vibration, vol. 313, no. 1-2, pp. 342-359, 2008.

[16] F. Luisier, T. Blu, and M. Unser, "A new SURE approach to image denoising: Interscale orthonormal wavelet thresholding," IEEE Transactions on Image Processing, vol. 16, no. 3, pp. 593-606, 2007.

[17] J. Liu, and P. Moulin, "Information-theoretic analysis of interscale and intrascale dependencies between image wavelet coefficients," IEEE Transactions on Image Processing, vol. 10, no. 10, pp. 1647$1658,2001$.

[18] L. Sendur, and I. Selesnick, "Bivariate shrinkage functions for wavelet-based denoising exploiting interscale dependency," IEEE Transactions on Signal Processing, vol. 50, no. 11, pp. 2744-2756, 2002.

[19] A. Pizurica, and W. Philips, "Estimating the probability of the presence of a signal of interest in multiresolution single- and multiband image denoising," IEEE Transactions on Image Processing, vol. 15, no. 3, pp. 654-665, 2006.

[20] M. Crouse, R. Nowak, and R. Baraniuk, "Wavelet-based statistical signal processing using hidden markov models," IEEE Transactions on Signal Processing, vol. 46, no. 4, pp. 886-902, 1998.

[21] T. Koshy, Discrete Mathematics with Applications. USA, Elsevier Academic Press, 2004.

[22] A. Antoniadis, J. Bigot, and T. Sapatinas, "Wavelet denoising. Procedures in Matlab," (Nov. 2009) Available at: http://wwwlmc.imag.fr/SMS/software/GaussianWaveDen/index.html

A. Antoniadis, J. Bigot, and T. Sapatinas, "Wavelet estimators in
nonparametric regression: a comparative simulation study," Journal of Statistical Software, vol. 6, no. 6, pp. 1-83, 2001,
http://www-lmc.imag.fr/lmc-sms/Anestis.Antoniadis/HTTP/publisA. Antoniadis, "Wavelet methods in statistics: Some recent developements and their applications," Statistics Surveys, vol. 1, S. Mallat, A Wavelet Tour of Signal Processing. Landon, Academic T. Cai, and B. Silverman, "Incorporating information on neighbouring coefficients into wavelet estimation," Sankhy $\bar{a}:$ The Indian Journal of Statistics. Special issue on Wavelets, vol. 63, no.
2, pp. 127-148, 2001.
R. Ranta, C. Heinrich, V. Louis-Dorr, and D. Wolf, "Interpretation and improvement of an iterative wavelet-based denoising method," R. Ranta, V. Louis-Dorr, C. Heinrich, and D. Wolf, "Iterative wavelet-based denoising methods and robust outlier detection," J.-L. Starck, and F. Murtagh, "Image restoration using wavelet transform," Astronomy and Astrophysics, vol. 288, pp. 342-348, 1994.

wavelet de-noising for medical signals and images," in TimeFrequency and Wavelets in Biomedical Engineering, M. Akay, Ed. L. Hadjileontiadis, L. Liatsos, C. Mavrogiannis, T. Rokkas, and S.
Panas, "Enhancement of bowel sounds by wavelet-based filtering," IEEE Transactions on Biomedical Enginering, vol. 47, no. 7, pp. 876-886, 2000.

for curve estimation using kernel and wavelet methods," The Annals of Statistics, vol. 26, no. 3, pp. 922-942, 1998

where $u$ is the estimated shape parameter of the GG

$$
p_{\sigma, u}(w)=\alpha e^{-|\beta w|^{u}} \text { with }
$$
the scale) or scale by scale, to deal with colored noise / signal (see cited references $[1,5,8]$ for details).

\section{REFERENCES}
[1] D. Donoho, and I. Johnstone, "Ideal spatial adaptation via wavelet shrinkage," Biometrika, vol. 81, pp. 425-455, 1994.
[2] D.L. Donoho, and I.M. Johnstone, "Adapting to unknown smoothness via wavelet shrinkage," Journal of the American Statistical Association, vol. 90, pp. 1200-1224, 1995.

Received: December 03, 2009

Revised: December 30, 2009

Accepted: January 02, 2010

(C) Ranta and Louis-Dorr; Licensee Bentham Open.

This is an open access article licensed under the terms of the Creative Commons Attribution Non-Commercial License (http://creativecommons.org/licenses/by-nc/3.0/) which permits unrestricted, non-commercial use, distribution and reproduction in any medium, provided the work is properly cited. 